\title{
Cystic fibrosis: transition from paediatric to adult physician's
} \section{care}

Advances in treatment have resulted in considerable improvements in prognosis for those with cystic fibrosis over the past 30 years. When first recognised, more than $80 \%$ of children died within the first two years, whereas now it is expected that most will survive to adult life with the median age of survival currently in the early $30 \mathrm{~s}$. It is estimated that at least half of those with cystic fibrosis will be adults by the year $2000 .^{1}$ It is difficult to identify specifically those interventions which have resulted in this change. Some programmes, such as mist tents and mucolytics, were readily accepted until properly controlled trials demonstrated no significant benefit. Others such as positive approach to management, early aggressive antibiotic therapy, high calorie diet, and improved enzymes appear to have contributed to the improved quality of life and prognosis. Physiotherapy has been shown to be of value ${ }^{2}$ but there remains considerable discussion regarding the effectiveness of different techniques including traditional postural drainage and percussion, forced expiratory technique, positive expiratory pressure masks, autodrainage, and flutter valves. Newer treatments which may or may not contribute to improvements in pulmonary status include DNase, non-steroidal anti-inflammatory agents, amiloride and uridine triphosphate, and immunisation with various components of the pseudomonas. Gene therapy is the most likely intervention to result in a dramatic change in long term outcome. Transfer of the ability to produce the cystic fibrosis transmembrane regulator has been demonstrated, but it will probably be many years before the technique is developed to a level which will provide clinical effectiveness with safety. In the meantime, heart/lung or double lung transplantation is the only option for prolonging life and improving the quality of life in those with terminal respiratory disease.

These changes highlight the importance of cystic fibrosis as a condition of adults as much as one of children. There are many critical periods in the life of an individual with cystic fibrosis which require special attention to ensure that both they and their families cope effectively. These include the time of diagnosis, entering school, adolescence, transition from paediatric to adult care, commencing employment, marriage and family, lung transplantation, and the terminal illness. Throughout the world, physicians in internal medicine and the relevant specialist clinical disciplines have developed an interest in cystic fibrosis so that there is now the opportunity for appropriate transfer from paediatric to adult physician. It has been well documented that children who have access to multidisciplinary teams in cystic fibrosis centres do better than those without such access. Although not confirmed, it would seem most likely that adults with cystic fibrosis would require the same resources to maintain the best quality of life.

Cystic fibrosis is a highly variable condition and each patient must be treated individually. Although the gene has been identified on the long arm of chromosome 7 and the $\Delta \mathrm{F} 508$ mutation is responsible for the majority in those of Central European descent, more than 400 other mutations have been identified. Although a strict genotype/phenotype correlation has not been defined, it is clear that various mutations have different effects in the production of the gene product, the cystic fibrosis transmembrane regulator. In some the protein is not made, in others a defective protein is made, the protein may not leave the endoplasmic reticulum, or it may not be processed. For most, the resulting outcome is increased susceptibility to respiratory infections leading to progressive lung damage and eventually respiratory failure. However, some do have a milder form of disease with minimal symptoms through childhood. This group is being increasingly recognised through neonatal screening using immunoreactive trypsin and through diagnosis by genetic studies. Many of these patients have pancreatic sufficiency. A considerable number will present a pattern of disease in adult life that has not been recognised in the past as being due to cystic fibrosis.

Lung pathology is the main cause of disease which affects lifestyle and prognosis. Although the lungs are normal at birth, abnormalities develop with the onset of the first infection and recent studies have suggested that this may occur asymptomatically in early infancy. Treatment through childhood aims at preventing this vicious cycle developing by aggressive early intervention. Failure to treat these infective processes effectively leads to progressive lung disease with bronchiectasis, haemoptysis, pneumothorax, and cor pulmonale. Fortunately, many of these complications are no longer present in adolescents who transfer to adult care. Those with pancreatic insufficiency can maintain good body mass index with effective enzymes and high calorie diets. Most adolescents are encouraged to develop an active exercise programme which should be maintained on transfer to an adult centre. Unfortunately, some will have failed to do so or have actively rebelled against such a programme. This appears to be more common in girls. Attempts to encourage such a programme remains essential during adult care, even if it has not been successful during paediatric care.

Distal intestinal obstruction syndrome with abdominal pain and progressive liver disease may be manifest around the time of transfer and will need careful attention. Ursodeoxycholic acid has been used for those with liver disease but its benefit has yet to be defined. Diabetes mellitus will also develop in up to $5 \%$ of young adults at this time. A small group will have significant problems with sinusitis and nasal polyps. Topical corticosteroids and surgery help but the condition tends to recur and cause considerable ongoing discomfort. A few patients will have significant joint disease, occasionally as a manifestation of hypertrophic osteoarthropathy, but in most it will be a nonspecific episodic arthritis associated with exacerbation of infection.

Psychosocial problems are considerable and certainly need to be dealt with at this critical period. However, in spite of the burden of illness in cystic fibrosis, psychological function in many respects matches well people. ${ }^{3}$ In this milieu, problems associated with the transfer from paediatric to adult care may relate to patient attitudes, family attitudes, and those of health care professionals. The patient needs to overcome a dependence on family and health care professionals if this has not been dealt with in the years leading up to transfer. The level of maturity will vary at different chronological ages in individuals. Attempts should be made for transfer to occur with development of maturity, but other factors may mitigate against this happening. Those patients who are severely ill, those with considerable psychopathology, and those who lack ap- 
propriate support systems will face greater difficulties with transfer.

The role of the family, and in particular of the parents, is likely to change dramatically from one of control and dependence to that of greater independence and decreased input into decision making with transfer. The effect will depend critically on the family's perceptions of the patient and the disease. Many families have a feeling of betrayal, of being dumped, after years of daily commitment to maintaining good health.

The paediatrician's attitude to transfer may be tempered by economics and a loss of a patient base for research. Continuing care over many years produces emotional ties with the patient and family that are difficult to break. His/ her attitude will be influenced by the perception of disease, the perception of their own skills and, in some cases, a distrust of adult caregivers. Some paediatricians have a fatalistic attitude at this age related to a lack of hope of long term survival due to poor understanding of the changing natural history of the disease. Paediatric health care professionals may fail to treat adult patients as such, and may be totally unaware of many subtle differences. They may not be comfortable discussing alcohol intake, sexual activity, and menstrual disturbances.

General physicians may have difficulties in coping with a patient who has a congenital abnormality as few of their other patients will have been adapting to a disease from birth. Some may have inadequate insight into the family dynamics. Until they develop a large population base, they may have poor knowledge of the diverse manifestations of the disease. There may be a lack of institutional commitment to management of adults with cystic fibrosis as they will constitute a significant economic burden costing up to $£ 20000$ per year per patient. ${ }^{4}$

Some patients with cystic fibrosis will be more severely ill at the time of transfer and each has to be dealt with individually. For some, that transfer may be beneficial and give renewed hope, while others may see death as inevitable as they may be aware of some who have died shortly after transfer. Both paediatrician and general physician need to be aware of these fears and perceptions. To cope with those who are severely ill they must be adept at dealing with imminent death and grieving of family and friends. The way in which it is dealt with will have an effect on all other patients, families, and health professionals in both centres.

Adolescence is a time of developing independence and a time of rebellion. In many, this may be associated with reduced compliance with treatment and substance abuse. Cigarette smoking has been associated with poorer clinical status. ${ }^{5}$ The general physician may cope better with smoking, alcohol intake, and illegal drug usage. The way in which these problems are dealt with around the time of transfer needs to be well coordinated and sensitively approached.

To be suitable for transfer, adult centres must involve a range of health professionals able to deal with the evolving problems in this condition at this age, including obstetrics and gynaecology, endocrinology, and psychiatry, as well as a respiratory physician and gastroenterologist. Conditions such as diabetes, respiratory failure and possible lung transplantation, as well as the increased risk of gastrointestinal cancer in the long term, may require additional specialist input. It will be important to deal with sexuality, family and career planning. Those responsible for paediatric care may fail to prepare their patients adequately. They should be helping to promote career decision making from an early age. Unfortunately, some children with cystic fibrosis are acutely aware that, unlike other children, they are rarely asked what they will do when they grow up.
Changes in the various health care systems around the world are resulting in programmes that may be disadvantageous to those with chronic illnesses including cystic fibrosis. Case mix funding may encourage shorter stays in hospital and this will not necessarily be optimal for some patients. Health maintenance organisations may insist on primary care being provided by a general physician. Insurance companies and bureaucrats will be more interested in the dollar cost of care than in the needs of the individual patient. Some insurance companies may drop children from the parent's policy at the time of transfer leading to considerable stress for all involved.

Although some have felt that the care of those with congenital abnormalities should remain within the province of a paediatric centre, most now accept that transfer to adult physicians and health carers is in the best interests of the patient. The effectiveness of transfer can be optimised by starting to make preparations well before it is due to take place. Between 12 and 15 years children should be seen first without parents so that both become used to accepting some degree of independence. The time of transfer will vary depending on the individuals. Most allow some flexibility between the ages of 15 and 19 years depending on the maturity, the patient's wishes, and the parental support system.

A transitional clinic where the patients are seen by the paediatrician and general physician together has been found to be effective. Visits to the adult centre before transfer may be helpful and booklets and other sources of information may contribute to a smooth transition. Continued communication between the paediatric and adult centres will give patients and their families confidence that the process is planned and monitored. There should be early transfer of information about patients from the paediatric centre with ongoing communication and effective feedback from the adult centre once transfer has occurred. Support groups involving young adults can assist the process. Clinics for young adults at times that do not interfere with school, university, or work schedules may be important for the young adult. The offer of home support, particularly if there is a planned move from the parental home, may be helpful for some. A survey of those who have transferred from a paediatric to an adult centre found that $81 \%$ liked to see the adult physician at the paediatric hospital and $64 \%$ found written material helpful. ${ }^{6}$

Those with cystic fibrosis and their families will have greater confidence in transfer if a transition programme is available. The personal introduction of new carers, resource material, and support groups will help to improve the effectiveness of this important process for those with a complex disease associated with significant physical, emotional, and social problems occurring at the same time.

Department of Paediatrics,

LOUIS I LANDAU

Princess Margaret Hospital for Children,

The Children's Hospital Medical Centre,

GPO Box D184,

Perth,

Western Australia 6001

1 Elborn JS, Shale D, Britton JR. Cystic fibrosis: current survival and population estimates to the year 2000. Thorax 1991;46:881-5.

2 Reisman JJ, Rivington-Law B, Corey M, Marcotte J, Wannamaker E, Harcourt $\mathrm{D}$, et al. Role of conventional physiotherapy in cystic fibrosis. $\mathcal{J}$ Pediatr 1988;113:632-6.

3 Blair C, Cull A, Freeman CP. Psychological functioning of young adults with cystic fibrosis and their families. Thorax 1994;49:798-802.

4 Robson M, Abbott J, Webb K, Dodd M, Walsworth-Bell J. A cost description of an adult cystic fibrosis unit and cost analyses of different categories of patients. Thorax 1992;47:684-9.

5 Campbell PW, Parker RA, Roberts BT, Krishnamani MR, Phillips JA III Association of poor clinical status and heavy exposure to tobacco smoke in patients with cystic fibrosis who are homozygous for F508 deletion. $\mathscr{F}$ Pediatr 1992;120:261-4.

6 Abdale B, Kuhl K, Tullis DE. Evaluation of patient satisfaction with the transition from a paediatric hospital to an adult centre (abstract). Pediat Pulmonol 1994;Suppl 10:291. 